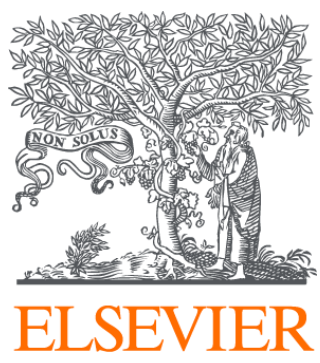

Since January 2020 Elsevier has created a COVID-19 resource centre with free information in English and Mandarin on the novel coronavirus COVID-

19. The COVID-19 resource centre is hosted on Elsevier Connect, the company's public news and information website.

Elsevier hereby grants permission to make all its COVID-19-related research that is available on the COVID-19 resource centre - including this research content - immediately available in PubMed Central and other publicly funded repositories, such as the WHO COVID database with rights for unrestricted research re-use and analyses in any form or by any means with acknowledgement of the original source. These permissions are granted for free by Elsevier for as long as the COVID-19 resource centre remains active. 


\title{
Traceable surveillance and genetic diversity analysis of coronaviruses in poultry from China in 2019
}

\author{
Yang Li ${ }^{\text {a, }}$, Qingye Zhuang ${ }^{\text {a, }}$, Lijian Jiang ${ }^{a}$, Wenming Jiang ${ }^{\text {a }}$, Cheng Peng ${ }^{a}$, Nan Jiang ${ }^{a}$, \\ Fuyou Zhang ${ }^{a}$, Xiaohui Yu ${ }^{a}$, Suchun Wang ${ }^{a}$, Liping Yuan ${ }^{a}$, Guangyu Hou ${ }^{a}$, Shuo Liu ${ }^{a}$, \\ Jingjing Wang ${ }^{a}$, Jianmin $\mathrm{Yu}^{\mathrm{a}}$, Jinping $\mathrm{Li}^{\mathrm{a}}$, Chenglong Zhao ${ }^{\mathrm{a}}$, Baoxu Huang ${ }^{\mathrm{a}}$, Hualei Liu ${ }^{\text {a,* }}$ \\ Kaicheng Wang ${ }^{\mathrm{a}, \mathrm{b}}$,"
}

${ }^{a}$ China Animal Health and Epidemiology Center, Qingdao, Shandong, China

${ }^{\mathrm{b}}$ Yebio Bioengineering Co., Ltd of Qingdao, Qingdao, Shandong, China

\section{A R T I C L E I N F O}

\section{Keywords:}

SARS-CoV-2

Avian-origin coronaviruses

Surveillance

Diversity

Cross-species transmission

\begin{abstract}
A B S T R A C T
Coronavirus disease 2019 (COVID-19), caused by Severe acute respiratory syndrome coronavirus 2 (SARS-CoV-2), was first reported in Wuhan, China, and rapidly spread throughout the world. This newly emerging pathogen is highly transmittable and can cause fatal disease. More than 35 million cases have been confirmed, with a fatality rate of about $2.9 \%$ to October 9,2020 . However, the original and intermediate hosts of SARS-CoV-2 remain unknown. Here, 3160 poultry samples collected from 14 provinces of China between September and December 2019 were tested for SARS-CoV-2 infection. All the samples were SARS-CoV-2 negative, but 593 avian coronaviruses were detected, including 485 avian infectious bronchitis viruses, 72 duck coronaviruses, and 36 pigeon coronaviruses, with positivity rates of $15.35 \%, 2.28 \%$, and $1.14 \%$, respectively. Our surveillance demonstrates the diversity of avian coronaviruses in China, with higher prevalence rates in some regions. Furthermore, the possibility that SARS-CoV-2 originated from a known avian-origin coronavirus can be preliminarily ruled out. More surveillance of and research into avian coronaviruses are required to better understand the diversity, distribution, cross-species transmission, and clinical significance of these viruses.
\end{abstract}

\section{Introduction}

Coronaviruses (CoVs) belong to the order Nidovirales, the family Coronaviridae, and the subfamily Orthocoronavirinae, and are divided into four genera: Alphacoronavirus, Betacoronavirus, Gammacoronavirus, and Deltacoronavirus (King et al., 2012). They can infect many animals, including humans (Cheng et al., 2017; Guan et al., 2003; Rota et al., 2003; Woo et al., 2006, 2009; Marra et al., 2003). Among these genera, Alphacoronavirus mainly infects humans, pigs, dogs, cats, bats, etc.; Betacoronavirus mainly infects humans, cattle, horses, pigs, mice, bats, and other mammals; Gammacoronavirus mainly infects domestic poultry; and Deltacoronavirus mainly infects wild birds and pigs. CoVs that can infect birds mainly belong to the genera Gammacoronavirus and Deltacoronavirus within the family Coronaviridae (King et al., 2011; Jordan et al., 2015). CoVs isolated from domestic poultry, such as Avian infectious bronchitis virus (IBV), belong to Gammacoronavirus (King et al.,
2012). Among the avian CoVs, IBV is most harmful to the poultry industry and is listed as a notifiable disease by the World Organization for Animal Health (OIE). Duck coronavirus (DuCoV), goose coronavirus, and pigeon coronavirus ( $\mathrm{PiCoV}$ ) have also been detected and are very genetically different from the IBVs (Chen et al., 2013; Jonassen et al., 2005; Zhuang et al., 2020).

In December 2019, an outbreak of an unknown pneumonia occurred in Wuhan, China (Zhou et al., 2020). The pathogen was soon identified as an emerging coronavirus, designated Severe acute respiratory syndrome coronavirus 2 (SARS-CoV-2) by the International Committee on the Taxonomy of Viruses (ICTV), and the disease was designated 'coronavirus disease 2019' (COVID-19) by the World Health Organization (WHO) (Chen et al., 2020). The clinical symptoms of COVID-19 predominantly include asymptomatic infection, mild-to-severe respiratory tract illness, and even death (Huang et al., 2020). Compared with Severe acute respiratory syndrome coronavirus (SARS-CoV), SARS-CoV-2 has a

\footnotetext{
* Correspondence author at: China Animal Health and Epidemiology Center, 369 Nanjing Road, Qingdao, Shandong, China.

E-mail addresses: liuhualei@cahec.cn (H. Liu), wangkaicheng@cahec.cn (K. Wang).

1 These authors contributed equally to this article.
} 


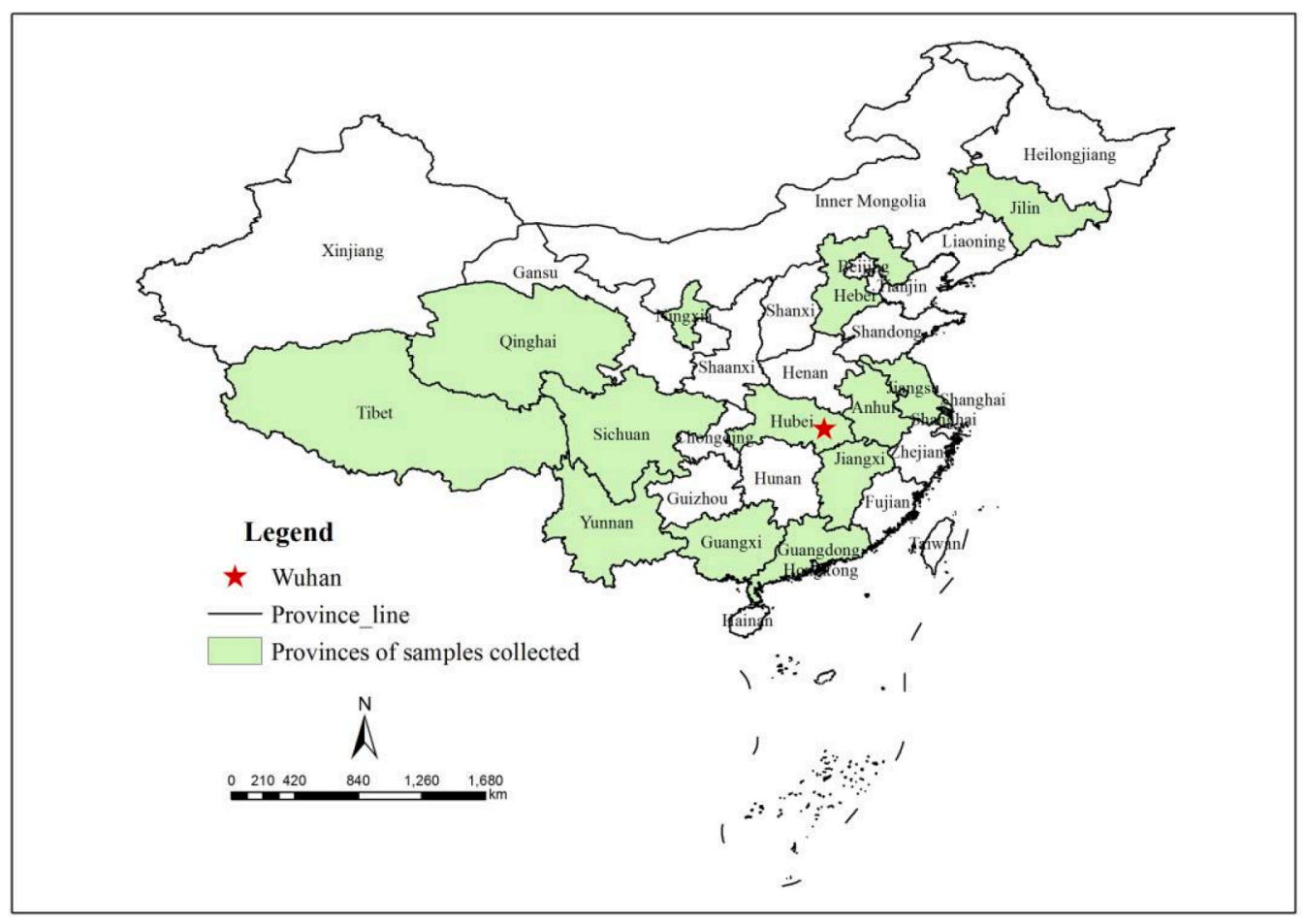

Fig. 1. Geographical distribution of the samples collected in 2019.

higher basic reproduction number, indicating greater transmissibility (Liu et al., 2020). Within a very short period of time, COVID-19 has quickly become a very serious threat to human health, travel, and commerce throughout the world (Stoecklin et al., 2020; Ghinai et al., 2020; Tuite et al., 2020).

The virus has been successfully isolated, but the pathogenic mechanism and effective vaccines are still undergoing extensive study. SARSCoV-2 belongs to the genus Betacoronavirus, which also includes SARS$\mathrm{CoV}$ and Middle East respiratory syndrome coronavirus (MERS-CoV). The natural host of the highly pathogenic SARS and MERS coronaviruses has been confirmed as bats, and recombination between bat and pangolin coronaviruses may represent to the origin of SARS-CoV-2 (Zhu et al., 2020). The transmission of SARS-CoV-2 from bats to humans is suspected to have occurred via direct contact between humans and intermediate host animals (Guo et al., 2020). However, it remains unclear which animals are the intermediate hosts of SARS-CoV-2. A previous study demonstrated that SARS-CoV can infect ferrets and cats (Martina et al., 2003), implying that these hosts might also be susceptible to SARS-CoV-2. Because poultry are in very close contact with humans, it is very important to identify any possible source of SARS-CoV-2 in avian species, especially in outbreak areas.

In this study, traceable surveillance was conducted to identify the possibility that SARS-CoV-2 originated in poultry. Tracheal and cloacal swabs collected during the routine surveillance of avian diseases in 2019 were tested with real-time reverse transcription (RT-PCR), as recommended by the Chinese Center for Disease Control and Prevention (CDC) to detect SARS-CoV-2. A universal RT-PCR was developed by our laboratory to analyze and molecularly characterize coronaviruses detected in poultry with DNA sequencing. Our study demonstrates the genetic diversity of the avian CoVs and that no SARS-CoV-2 infection was present in poultry in China in 2019.

\section{Material and methods}

\subsection{Ethics statement}

This study was conducted according to the animal welfare guidelines of the World Organization for Animal Health (Thiermann, 2015) and was approved by the Animal Welfare Committee of the China Animal Health and Epidemiology Center.

\subsection{Sample collection}

A total of 3160 swab samples were collected in September-December 2019 in 14 provinces of China (including Hubei), mainly for the routine surveillance of avian diseases such as avian influenza viruses (AIVs), Newcastle disease virus (NDV), etc. The swab samples were collected by taking smears from both the cloacal and oropharyngeal tracts of each bird, and stored in $1.5 \mathrm{~mL}$ of phosphate-buffered saline ( $\mathrm{pH} 7.2$ ) containing $10 \%$ glycerol. The RNA was extracted with the RNeasy Mini Kit (Qiagen, Hilden, Germany), according to manufacturer's instructions, and stored at $-80{ }^{\circ} \mathrm{C}$.

\subsection{Nucleic acid detection of SARS-CoV-2}

All RNA samples were examined for SARS-CoV-2 nucleic acid using real-time RT-PCR based on the recommendations of the Chinese CDC (http://ivdc.chinacdc.cn / /kyjz/202,001/t20200121_211,337.html). Briefly, two target genes, the nucleoprotein $(\mathrm{N})$ gene and open reading frame (ORF) $1 \mathrm{ab}$, were simultaneously amplified and detected with a real-time RT-PCR assay. The primers used were Target $1(\mathrm{~N})$ : forward primer GGGGAACTTCTCCTGCTAGAAT, reverse primer CAGACATTTTGCTCTCAAGCTG, and probe $5^{\prime}$-FAM-TTGCTGCTGCTTGACAGATT-TAMRA-3'; and Target 2 (ORF1ab): forward primer CCCTGTGGGTTTTACACTTAA, reverse primer ACGATTGTGC ATCAGCTGA, and probe $5^{\prime}$-VIC-CCGTCTGCGGTATGTGGAAAGGTTATGG-BHQ1-3'. The criteria for a confirmed diagnosis of SARS-CoV-2 were the positive amplification of both genes, $\mathrm{N}$ and ORF1ab.

\subsection{Detection of COV nucleic acids with a conserved RT-PCR assay}

A conserved RT-PCR assay and DNA sequencing were used to analyze the genetic characteristics of the CoVs circulating in the poultry. In brief, the stored RNAs described above were amplified with the 


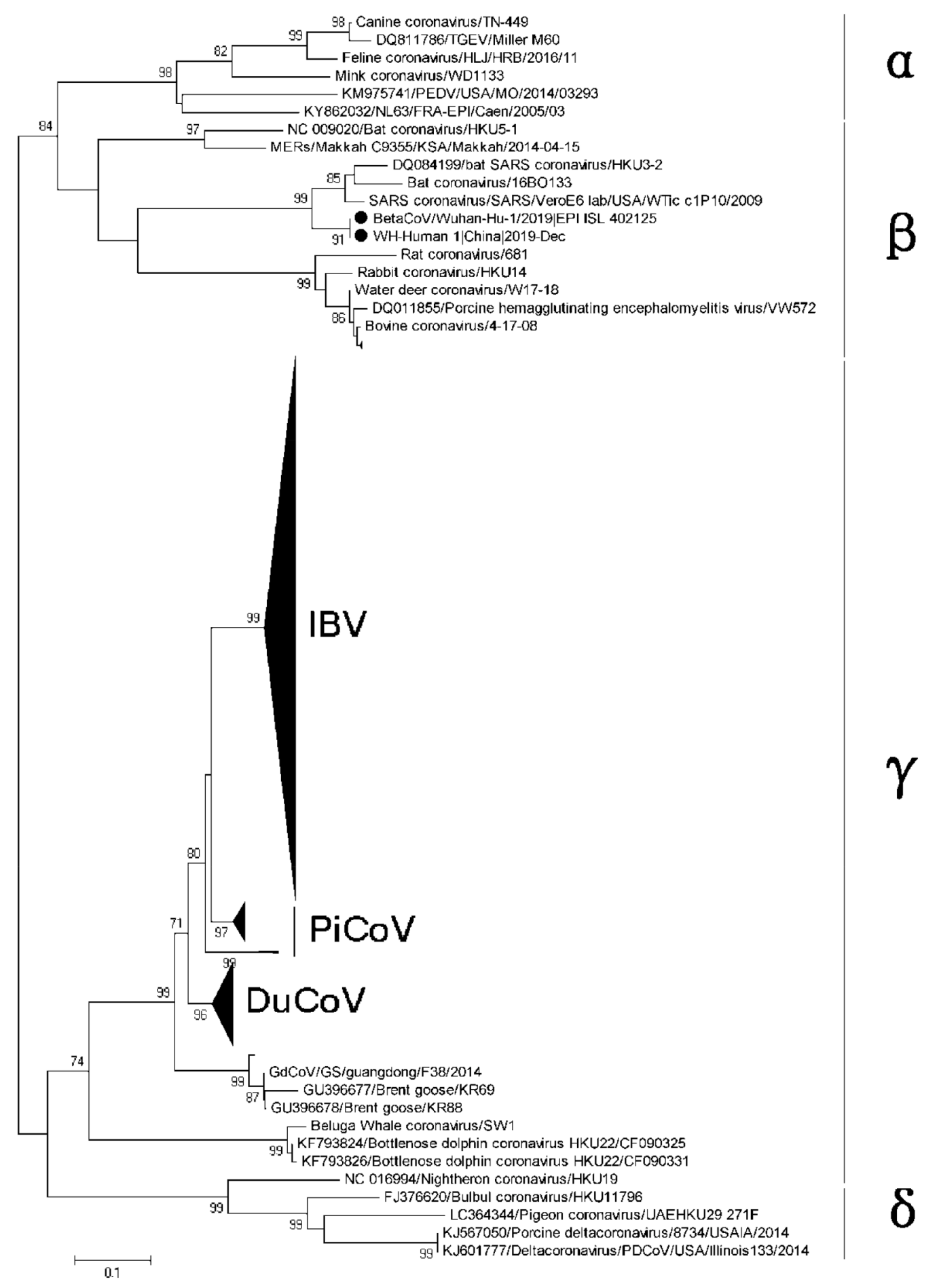

Fig. 2. Phylogenetic trees based on the RdRp gene sequences of coronaviruses. The trees were constructed using the model with the maximum likelihood (ML) method, gaps were handled by partial deletion and bootstrap values were calculated out of 1000 replicates. IBV: avian infectious bronchitis virus; DuCoV: duck coronavirus; PiCoV: pigeon coronavirus; SARS-CoV-2: severe acute respiratory syndrome coronavirus 2. Two SARS-CoV-2 representative strains were marked with black solid circles (-), which belonged to the Betacoronavirus of Coronaviridae. A total of 593 avian coronaviruses were detected, including 485 IBVs, 72 DuCoVs and 36 PiCoVs, which all belonged to the Gammacoronavirus.
Takara One Step RT-PCR Kit (Takara), using a conserved RT-PCR assay designed in our laboratory, with primers $5^{\prime}$-GGTTGGGATTAYCCWAARTGYGA-3' (forward) and 5'-YTGTGAACAAAAYTCRTGWGGACC$3^{\prime}$ (reverse). The amplify region is $14,181-14,780 \mathrm{bp}$ in the RDRP gene of the reference sequence (The GenBanK Accession number is NC_001451). The assay amplifies a 600-bp nucleotide region in the viral ORF1ab, and this assay has been shown to detect the main CoVs circulating in animals, including pigs, chickens, ducks, geese, and pigeons. The RT-PCR was performed in a $25 \mu \mathrm{l}$ reaction system with incubation at $50{ }^{\circ} \mathrm{C}$ for $30 \mathrm{~min}$, followed by denaturation at $94^{\circ} \mathrm{C}$ for $5 \mathrm{~min}$, and 30 cycles of $94{ }^{\circ} \mathrm{C}$ for $30 \mathrm{~s}, 50{ }^{\circ} \mathrm{C}$ for $30 \mathrm{~s}$, and $72{ }^{\circ} \mathrm{C}$ for $45 \mathrm{~s}$. The PCR products were purified with an agarose gel DNA extraction kit (Sangon,
Shanghai, China), and sequenced directly with the ABI 3730xl DNA Analyzer. The sequences were used in a subsequent phylogenetic analysis.

\subsection{Phylogenetic analysis}

The sequences were aligned and the substitution models and phylogenetic relationships were calculated with the software package MEGA 6.0 (Hall, 2013; Tamura et al., 2013). The phylogenetic relationships of the sequences were calculated with the maximum likelihood (ML) model, which is assumed to best describe the substitution pattern. The gaps were handled by partial deletion, and bootstrap values 
Table 1

Positive rates (\%) of three lineages of CoVs in the samples collected from four host species in 2019.

\begin{tabular}{lllll}
\hline & Chickens & Ducks & Geese & Pigeons \\
\hline IBV & $98.97 \%(480 /$ & $0.82 \%(4 / 485)$ & $0.21 \%(1 /$ & \\
& $485)$ & & $485)$ & \\
DuCoV & $12.50 \%(9 / 72)$ & $87.50 \%(63 /$ & & \\
& & $72)$ & & $77.78 \%(28 /$ \\
PiCoV & $22.22 \%(8 / 36)$ & & $36)$ \\
\hline
\end{tabular}

Table 2

Number of samples and positive rate of three lineages of CoVs in each province.

\begin{tabular}{lllllll}
\hline Province & $\begin{array}{l}\text { Number of } \\
\text { samples }\end{array}$ & DuCoV & IBV & PiCoV & Total & Rate \\
\hline Anhui & 308 & 5 & 44 & 5 & 54 & $17.53 \%$ \\
Guangdong & 267 & 9 & 12 & & 21 & $7.87 \%$ \\
Guangxi & 256 & 19 & 34 & 3 & 56 & $21.88 \%$ \\
Hebei & 256 & 6 & 42 & & 48 & $18.75 \%$ \\
Hubei & 326 & 18 & 100 & & 118 & $36.20 \%$ \\
Jilin & 79 & 3 & 3 & 4 & 7 & $8.86 \%$ \\
Jiangsu & 130 & 11 & 86 & 5 & 102 & $33.44 \%$ \\
Jiangxi & 305 & & 12 & 2 & 14 & $7.91 \%$ \\
Ningxia & 177 & & 29 & & 29 & $27.62 \%$ \\
Qinghai & 105 & & 16 & 7 & 23 & $14.38 \%$ \\
Shanghai & 160 & & 32 & 3 & 35 & $11.04 \%$ \\
Sichuan & 317 & & 52 & 3 & 55 & $24.77 \%$ \\
Xizang & 222 & 1 & 14 & & 15 & $5.95 \%$ \\
Yunnan & 252 & 72 & 485 & 36 & 593 & $18.77 \%$ \\
Total & 3160 & & & & &
\end{tabular}

were calculated from 1000 replications (Li, 1997).

\section{Results}

\subsection{Distribution of samples detected in 2019}

A total of 3160 swab samples were collected in 14 provinces of China in 2019 (Anhui, Guangdong, Guangxi, Hebei, Hubei, Jiangsu, Jiangxi, Jilin, Ningxia, Qinghai, Shanghai, Sichuan, Xizang, and Yunnan). The geographic distribution of the samples detected in this study is shown in Fig. 1. The samples included 2238 chicken samples, 669 duck samples, 184 pigeon samples, 59 geese samples, and 15 other avian samples (10 from wild geese, five from partridges), which originated in 48 retail markets, 18 wholesale markets, four slaughterhouses, and 18 poultry farms.

\subsection{Surveillance of SARS-CoV-2 in poultry}

The 3160 samples were tested with real-time RT-PCR, as recommended by China CDC, to detect SARS-CoV-2 nucleic acid, and all samples were negative for SARS-CoV-2.

\subsection{Surveillance of CoVs circulating in poultry}

Of the 3160 samples tested, $593 \mathrm{CoV}$-positive samples were detected with the conserved RT-PCR assay. The viral amplicons (ORF1ab fragments) were sequenced. The GenBank accession numbers for these $593 \mathrm{CoV}$ sequences are MT944357-MT944949.

\subsection{Phylogenetic and diversity analyses of CoVs circulating in poultry}

A phylogenetic analysis revealed that the 593 CoVs detected in poultry clustered into three lineages, corresponding to IBV $(n=485)$, DuCoV ( $n=72$ ), and PiCoV ( $n=36$ ) (Fig. 2). Emerging SARS-CoV-2 belongs to the genus Betacoronavirus in the family Coronaviridae, and the 593 avian CoVs detected in this study belonged to the genus Gammacoronavirus. SARS-CoV-2 was highly genetically distant from these avian CoVs.

The positivity rates for the IBVs, DuCoVs, and PiCoVs were $15.35 \%$, $2.28 \%$, and $1.14 \%$, respectively (Table 1 ). Our data suggest that IBVs mainly circulate in chickens, DuCoVs mainly circulate in ducks, and PiCoVs mainly circulate in pigeons. The number of positive samples and the rates of detection of the three lineages of CoVs in each province are shown in Table 2. IBV was detected in all provinces investigated in this study, and DuCoV and PiCoV were detected in most provinces. The province with the highest rate of CoVs was Hubei (36.20\%), and that with the lowest was Yunnan (5.95\%).

The numbers of due-species infections and cross-species infections in the samples collected from live poultry markets (LPMs) are shown in Table 3. Cross-species infections of CoVs (i.e., IBV infections in birds other than chickens, DuCoV infections in birds other than ducks, and PiCoV infections in birds other than pigeons) were identified in 23 of the 3160 samples from four sites (six involving IBVs, nine involving DuCoVs, and eight involving PiCoVs).

\section{Discussion}

So far, seven different human CoVs have been identified: SARS-CoV, MERS-CoV, human coronavirus NL63 (HCoV-NL63), HCoV-229E, HCoV-OC43, HCoV-HKU1, and SARS-CoV-2. Bats are deemed to be the natural hosts of SARS-CoV, MERS-CoV, HCoV-NL63, and HCoV$229 \mathrm{E}$, and rodents may be the natural hosts of HCoV-OC43 and HCoVHKU1 (Khan et al., 2020). The intermediate hosts of SARS-CoV, MERS-CoV, HCoV-229E, and HCoV-OC43 are palm civets, dromedary camels, alpacas, and cattle, respectively. However, the intermediate host (s) of SARS-CoV-2 remains unknown.

Because SARS-CoV-2 is genetically close to SARS-CoV, it has been suggested that bats are its natural host (Phan, 2020). A study showed that the common ancestor of SARS-CoV-2 and SARS-CoV was similar to the bat coronavirus HKU9-1 (Zhou et al., 2020), while Andersen et al. documented the possible natural origin of SARS-CoV-2 from BatCoV RaTG13 (Andersen et al., 2020). The pangolin is suspected to be a direct animal source of SARS-CoV-2 in humans because SARS-CoV-2-related CoVs have been isolated from Malayan pangolins and shared $97.4 \%$ similarity with SARS-CoV-2 in the receptor-binding domain in the viral S gene (Zhang et al., 2020).

Studies have shown that SARS-CoV-2 possibly originated from recombination between bat and pangolin coronaviruses (Zhu et al., 2020). To date, findings from experimental infection studies have suggested that poultry and pigs are not susceptible to SARS-CoV-2 infection (Shi et al., 2020). Of the animal species investigated, cats are the species most susceptible to SARS-CoV-2, and SARS-CoV-2 can be transmitted

Table 3

Numbers of due-species infections and cross-species infections in the samples collected from LPMs.

\begin{tabular}{|c|c|c|c|c|c|c|c|}
\hline & \multicolumn{3}{|c|}{ Due-species infections } & \multicolumn{3}{|c|}{ Cross-species infections } & \multirow[t]{2}{*}{ Rate } \\
\hline & IBV & DuCoV & $\mathrm{PiCoV}$ & IBV & DuCoV & $\mathrm{PiCoV}$ & \\
\hline retail markets $(n=1280)$ & 97 & 31 & 13 & 3 & 2 & 1 & $11.48 \%(147 / 1280)$ \\
\hline wholesale markets $(n=1495)$ & 320 & 32 & 15 & 3 & 1 & 1 & $24.82 \%(371 / 1495)$ \\
\hline $\operatorname{slaughterhouse}(n=245)$ & 57 & & & & 6 & & $25.71 \%(63 / 245)$ \\
\hline Poultry farms $(n=140)$ & 6 & & & & & 6 & $8.57 \%(12 / 140)$ \\
\hline
\end{tabular}


between cats by respiratory droplets (Jiang et al., 2012). In the laboratory setting, ferrets are susceptible to infection. The susceptibility of minks was documented by a report from the Netherlands of an outbreak of SARS-CoV-2 infection in farmed minks (Oreshkova et al., 2020). Golden Syrian hamsters, as well as cynomolgus and rhesus macaques, can be consistently infected with SARS-CoV-2 and may show clinical signs. Dogs also appear to be susceptible to infection, but appear to be less affected than ferrets or cats. Both virological and serological testing found evidence for natural SARS-CoV-2 infections in two dogs from households with human cases of COVID-19 in Hong Kong (Sit et al., 2020). Also, lions, pumas, and tigers at zoos, all belonging to the same family as cats (Felidae), were infected by asymptomatic and symptomatic patients in two countries (USA and South Africa), with SARS-CoV-2 detected in their fecal samples. In addition to pet and zoo animals, 138 infected mink farms were reported in 11 countries, with about $90 \%$ in Europe and 10\% in North America (Jia et al., 2021).

Our data show that all the samples collected from domestic poultry in 14 provinces of China (including Hubei) in 2019 were negative for SARS-CoV-2. Shi et al. also found that poultry, such as chickens and ducks, were not susceptible to SARS-CoV-2 (Shi et al., 2020), which is consistent with our results.

We also conducted a large-scale surveillance of avian CoVs in 3160 samples using a conserved RT-PCR assay, which detected avian CoVs such as IBVs, DuCoVs, and PiCoVs. Our results demonstrate that IBVs, DuCoVs, and PiCoVs belong to distinct lineages, although they all belong to the genus Gammacoronavirus in the family Coronaviridae, whereas SARS-CoV-2 belongs to the genus Betacoronavirus. SARS-CoV-2 was highly genetically distant from the avian CoVs.

IBV was detected in all the provinces investigated in this study, and DuCoV and PiCoV were detected in most provinces. IBV presents a risk to the poultry industry and is listed as a notifiable disease by the OIE, but the pathogenicity of DuCoV and PiCoV in poultry is still unclear, and warrants further research. The positive rates of IBV, DuCoV, and PiCoV infections were $15.35 \%, 2.28 \%$, and $1.14 \%$, respectively. These CoVs are also highly prevalent in LPMs, and our results also suggest their high prevalence in slaughterhouses. Therefore, the sites of poultry breeding, marketing, and slaughter (poultry farms, LPMs, and slaughterhouses) probably play important roles in the circulation of CoVs in poultry, as they do in the circulation of AIVs (Jiang et al., 2012).

Coronaviruses clearly have the capacity to jump species boundaries and adapt to new hosts, making it straightforward to predict that more will emerge in the future, although quite why coronaviruses possess this capacity in comparison to some other RNA viruses is unclear. Critically, the surveillance of animal coronaviruses should include animals other than bats, as the role of intermediate hosts is likely of major importance, providing a more direct pathway for the virus to emerge in humans (Zhang et al., 2020). While our intimate relationship with the animal world means we cannot build impregnable barriers, stronger action against the illegal wildlife trade and removing all mammalian (and perhaps avian) wildlife from wet markets will provide an important buffer.

In conclusion, the possibility that SARS-CoV-2 originated from a known avian-origin CoV can be preliminarily ruled out according to our analysis. However, the continuous surveillance of animal-origin CoVs should be increased to better understand the diversity, distribution, cross-species transmission, and clinical significance of CoVs in nature.

\section{Author statement}

Yang Li and Kaicheng Wang contributed to the design of the experimental work and the bioinformatics analysis. Hualei Liu revised the manuscript. Qingye Zhuang and Su-chun Wang contributed to the experimental work and drafting the manuscript. Baoxu Huang, Lijian Jiang, Wenming Jiang, Cheng Peng, Nan Jiang, Fuyou Zhang and Xiaohui Yu revised the manuscript. Suchun Wang, Liping Yuan and Guangyu Hou performed the bioinformatics analysis. Shuo Liu, Jingjing
Wang, Jianmin Yu, Jinping Li and Chenglong Zhao performed experimental work. All authors reviewed the manuscript.

\section{Declaration of Competing Interest}

The authors declare that they have no known competing financial interests or personal relationships that could have appeared to influence the work reported in this paper.

\section{Acknowledgements}

This work was supported by the National Key Research and Development Program of China (2020YFC0840800 and 2017YFC120050).

\section{References}

Andersen, K.G., Rambaut, A., Lipkin, W.I., et al., 2020. The proximal origin of SARS-CoV2. Nat. Med. 26 (4), 450-452. https://doi.org/10.1038/s41591-0820-9.

Chen, G.Q., Zhuang, Q.Y., Wang, K.C., et al., 2013. Identification and survey of a novel avian coronavirus in ducks. PLoS ONE 8 (8), e72918. https://doi.org/10.1371/ journal.pone.0072918.

Chen, N.S., Zhou, M., Dong, X., et al., 2020. Epidemiological and clinical characteristics of 99 cases of 2019 novel coronavirus pneumonia in Wuhan, China: a descriptive study. Lancet 395, 507-513. https://doi.org/10.1016/S0140-6736(20)30211-7.

Cheng, V.C., Lau, S.K., Woo, P.C., et al., 2017. Severe acute respiratory syndrome coronavirus as an agent of emerging and reemerging infection. Clin. Microbiol. Rev. 20, 660-694. https://doi.org/10.1128/CMR.00023-07.

Ghinai, I., McPherson, T.D., Hunter, J.C., et al., 2020. First known person-to-person transmission of severe acute respiratory syndrome coronavirus 2 (SARS-CoV-2) in the USA. Lancet 395, 1137-1144. https://doi.org/10.1016/S0140-6736(20)306073.

Guan, Y., Zheng, B.J., He, Y.Q., et al., 2003. Isolation and characterization of viruses related to the SARS coronavirus from animals in southern China. Science 302 , 276-278. https://doi.org/10.1126/science.1087139.

Guo, Y.R., Cao, Q.D., Hong, Z.S., et al., 2020. The origin, transmission and clinical therapies on coronavirus disease 2019 (COVID-19) outbreak-an update on the status. Mil. Med. Res. 7 (1) https://doi.org/10.1186/s40779-020-00240-0.

Hall, B.G., 2013. Building phylogenetic trees from molecular data with MEGA. Mol. Biol. Evol. 30 (5), 1229-1235. https://doi.org/10.1093/molbev/mst012.

Huang, C.L., Wang, Y.M., Li, X.W., et al., 2020. Clinical features of patients infected with 2019 novel coronavirus in Wuhan, China. Lancet 395, 497-506. https://doi.org/ 10.1016/S0140-6736(20)30183-5.

Jia, P., Dai, S., Wu, T., et al., 2021. New approaches to anticipate the risk of reverse zoonosis. Trends Ecol. Evol. (Amst.) 36 (7), 580-590. https://doi.org/10.1016/j. tree.2021.03.012.

Jiang, W.M., Liu, S., Hou, G.Y., et al., 2012. Chinese and global distribution of H9 subtype avian influenza viruses. PLoS ONE 7 (12), e52671. https://doi.org/10.1371/ journal.pone.0052671.

Jonassen, C.M., Kofstad, T., Larsen, I.L., et al., 2005. Molecular identification and characterization of novel coronaviruses infecting graylag geese (Anser anser), feral pigeons (Columbia livia) and mallards (Anas platyrhynchos). J. Gen. Virol. 86 (6), 1597-1607. https://doi.org/10.1099/vir.0.80927-0.

Jordan, B.J., Hilt, D.A., Poulson, R., et al., 2015. Identification of avian coronavirus in wild aquatic birds of the central and eastern USA. J. Wildl. Dis. 51, 218-221. https:// doi.org/10.7589/2014- 03-070.

Khan, S., Siddique, R., Shereen, M.A., et al., 2020. The emergence of a novel coronavirus (SARS-CoV-2), their biology and therapeutic options. J. Clin. Microbiol. 58 (5) https://doi.org/10.1128/JCM.00187-20.

King, A.A.Q., Adams, M.J., Carstens, E.B., 2012. Virus taxonomy: Classification and Nomenclature of viruses: Ninth Report of the International Committee On Taxonomy of Viruses, 806-828. Elsevier Academic Press, San Diego, p. 1276. https://doi.org/ 10.1159/000149278.

King, A.M.Q., Carstens, E.B., Lefkowitz, E.J, 2011. Ninth report of the international committee on taxonomy of viruses. Paramyxoviridae. 329-426.

Li, W.H., 1997. Molecular Evolution. Sinauer Associates Inc., Sunderland, pp. 149-175. https://doi.org/10.1371/journal.pone.0052671.

Liu, Y., Gayle, A.A., Wilder-Smith, A., et al., 2020. The reproductive number of COVID19 is higher compared to SARS coronavirus. J. Travel Med. 27 https://doi.org/ 10.1093/jtm/taaa021.

Marra, M.A., Jones, S.J.M., Astell, C.R., et al., 2003. The Genome sequence of the SARSassociated coronavirus. Science 2003 (300), 1399-1404. https://doi.org/10.1126/ science. 1085953.

Martina, B.E., Haagmans, B.L., Kuiken, T., et al., 2003. Virology: SARS virus infection of cats and ferrets. Nature 425 (6961), 915. https://doi.org/10.1038/425915a.

Oreshkova, N., Molenaar, R.J., Vreman, S., et al., 2020. SARS-CoV-2 infection in farmed minks, the Netherlands, April and May 2020. Eurosurvllance 25 (23). https://doi. org/10.2807/1560-7917.ES.2020.25.23.2001 005.

Phan, T., 2020. Genetic diversity and evolution of SARS-CoV-2. Infect., Genet. Evol. 81, 104260 https://doi.org/10.1016/j.meegid. 2020. 104260. 
Rota, P.A., Oberste, M.S., Monroe, S.S., et al., 2003. Characterization of a novel coronavirus associated with severe acute respiratory syndrome. Science 300 , 1394-1399. https://doi.org/10.1126/science.10859 52.

Shi, J.Z., Wen, Z.Y., Zhong, G.X., et al., 2020. Susceptibility of ferrets, cats, dogs, and other domesticated animals to SARS-coronavirus 2. Science 368 (6494), 1016-1020. https://doi.org/10.1126/science.abb7015.

Sit, T.H.C., Brackman, C.J., Ip, S.M., et al., 2020. Infection of dogs with SARS-CoV-2. Nature 586, 776-778. https://doi.org/10.1038/s41586- 020- 2334-5.

Stoecklin, S.B., Rolland, P., Silue, Y., et al., 2020. First cases of coronavirus disease 2019 (COVID-19) in France: surveillance, investigations and control measures. Eurosurveillance 25 (6). https://doi.org/10.2807/1560-7917.ES.2020.25.6.2000 094.

Tamura, K., Stecher, G., Peterson, D., et al., 2013. MEGA6: molecular Evolutionary Genetics Analysis version 6.0. Mol. Biol. Evol. 30 (12), 2725-2729. https://doi.org/ 10.1093/molbev/mst197.

Thiermann, A.B., 2015. International standards: the world organisation for animal health terrestrial animal health code. Revue Scientifique Et Technique 34 (1), 277-281. https://doi.org/10.20506/rst.34.1.2340.

Tuite, A.R., Ng, V., Rees, E., et al. 2020. Estimation of COVID-19 outbreak size in Italy based on international case exportations. The Lancet infectious diseases. 20(5): 537-537. https://doi.org/10.1101/2020.03.02.20030049.
Woo, P.C.Y., Lau, S.K.P., Lam, C.S.F., et al., 2009. Comparative analysis of complete genome sequences of three avian coronaviruses reveals a novel group $3 \mathrm{c}$ coronavirus. J. Virol. 83, 908-917. https://doi.org/10.1128/JVI. 01977-08.

Woo, P.C.Y., Lau, S.K.P., Yip, C.C.Y., et al., 2006. Comparative analysis of 22 coronavirus HKU1 genomes reveals a novel genotype and evidence of natural recombination in coronavirus HKU1. J. Virol. 80, 7136-7145. https://doi.org/10.1128/JVI.00509-06.

Zhang, Z.P., Xiao, K.P., Zhang, X., et al., 2020. Emergence of SARS-like coronavirus in China: an update[J]. J. Infect. 80 (5), e28-e29. https://doi.org/10.1016/j. jinf.2020.03.010.

Zhou, P., Yang, X.L., Wang, X.G., et al., 2020. A pneumonia outbreak associated with a new coronavirus of probable bat origin. Nature 579, 270-273. https://doi.org/ 10.1038/s41586-020-2012-7.

Zhu, Z., Meng, K., Meng, G., 2020. Genomic recombination events may reveal the evolution of coronavirus and the origin of SARS-CoV-2. Sci. Rep. 10 (1), 1-10. https://doi.org/10.1038/s41598-020-78703-6.

Zhuang, Q.Y., Liu, S., Zhang, X.C., et al., 2020. Surveillance and taxonomic analysis of the coronavirus dominant in pigeons in China. Transbound. Emerg. Dis. 67 (5), 1981-1990. https://doi.org/10.1111/tbed.13541. 